\title{
Extrasolar planets
}

\author{
Jack J. Lissauer
}

Natural philosophers have speculated on the existence of worlds around other suns for millennia. Now that real data are available, we find a diversity far beyond that expected by scientists, or science-fiction writers.

n the 1960s, with great fanfare, the discovery of first one, and then two Jupiter-like planets in orbit around Barnard's star was announced (Fig. 1). Only 6 light years away — but still too faint to see with the unaided eye - Barnard's star is one of the Sun's nearest neighbours (only the Alpha Centauri system is closer). But by the 1970 s, the evidence for these purported planets was discredited. More claims of the discovery of the first extrasolar planet, or 'exoplanet', continued to capture newspaper headlines, but these too failed to stand up to scrutiny.

It was only after decades of false leads that, in 1991, two bona fide extrasolar planets were detected ${ }^{1}$, and this discovery has stood the test of time. Exoplanets are small, very faint objects, located close to much brighter stars. The planets themselves have not been seen, but instead they have been identified by the gravitational tugs that they exert on their stars.

About 100 exoplanets are now known; most are comparable in mass to Jupiter, and have orbital periods of a few years or less. Astronomers are amassing a variety of detection techniques to better assess the diversity of planetary systems within our Galaxy. And the hunt is on for a true analogue of our Solar System that has an Earth-like planet, perhaps harbouring life as we know it.

\section{What is a planet?}

Five planets, or 'wandering stars', were known to the ancients: Mercury, Venus, Mars, Jupiter and Saturn. The astronomical revolution brought about by Copernicus, Kepler and Newton showed that these objects were more akin to the Earth than to the Sun and other stars. Thus our home orb was added to the list of known planets. Then, in 1781, the scientific world was taken by surprise when amateur telescope-maker William Herschel announced the discovery of a more distant planet, subsequently named Uranus. In 1800, the small planetary object Ceres was discovered in orbit between Mars and Jupiter, and tens of thousands of even smaller minor planets - asteroids have since been detected in that region. The planet Neptune signalled its presence through its gravitational effect on the orbit of Uranus, and was first actually seen in 1846. And Pluto, the furthermost Solar System planet known to us, appeared in a careful optical search carried out by Clyde Tombaugh in 1930.
N A T U R E November 30, 1963

VOL. 200

\section{NEWS and VIEWS}

\section{Barnard's Star}

BARNARD's STAR is the second nearest known stellar system, with apparent visual magnitude $9 \cdot 5$, a parallax of $0^{\prime \prime} .55$ and a proper motion of $10^{\prime \prime} \cdot 3$ per annum. It has been investigated at the Sproul Observatory of Swarthmore College by P. van de Kamp (Astronomical J., 68, 515; 1963 ) on photographs taken over a period of forty-five years; 2,413 plates with 8,260 exposures obtained un 609 nights by many observers. Barnard's Star has the largest known secular perspective acceleration in proper motion. This was determined $\left(-0^{\prime \prime} \cdot 00014\right.$ in R.A., $+0^{\prime \prime} \cdot 00118$ in declination). The residuals of the observations from the predicted positions allowing for proper motion, secular acceleration and parallax wore investi. gated. The residuals showed a periodicity of about 24 years, and indicate the presence of a companion. A semi-axis major of $0^{\prime \prime} .0245$ and an eccentricity of 0.6 for the main star fit the observations. If the mass of Barnard's Star is 0.15 solar mass, the companion has an orbit with semi-axis major of $2^{\prime \prime} \cdot 4$, or 4.4 astronomical units, and from the displacements of the main star it can be shown that the mass of the companion is 0.0015 solar masses, or 1.6 times the mass of Jupiter. Its apparent visual magnitude would be perhaps 30 , far below the reach of existing telescopes.

Figure 1 The 1963 'discovery' of a planet in orbit around Barnard's star is reported in Nature. The image above shows the Cone nebula, a star-forming region 2,500 light years from Earth, whose clouds of gas and dust could one day form planets.

The search for more planetary companions to the Sun continues, using direct imaging as well as the indirect signature of gravitational perturbations of the motions of 

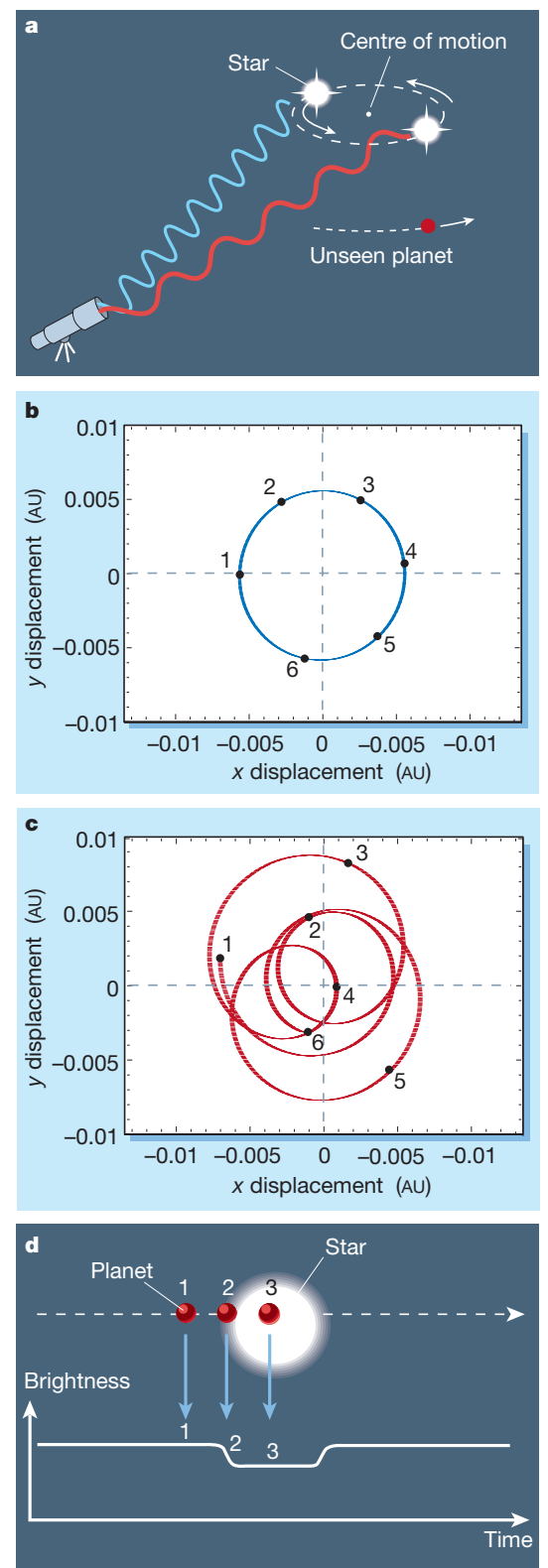

Figure 2 Signatures of exoplanets. a, The Doppler effect. An unseen planet influences the motion of its star: as the star moves towards the observer, the observed frequency of the starlight increases, becoming 'blueshifted'; as the star moves away, the light frequency decreases becomes 'redshifted'. By detecting these frequency changes, astronomers can infer the presence of the orbiting planet.

b, Astrometric data show the effect of Jupiter on the position of the Sun, and c, the combined effect of all nine Solar System planets. Points 1-6 indicate the position in years 2000-2050; 1 astronomical unit $(\mathrm{AU})$ is the mean distance between the Earth and the Sun. (Courtesy E. V. Quintana.) d, Partial eclipses of a star by an orbiting planet - or 'transits' - can be picked up through the distinctive dip that is periodically observed in the star's brightness. known planets, comets and even spacecraft.

Pluto was originally thought to be more massive than the Earth, but subsequent observations showed that it is less than $5 \%$ of the mass of Mercury, the smallest of the planets known before 1800 and itself less than $6 \%$ of the mass of the Earth. This realization, together with the discovery of many minor planets beyond Neptune during the past decade (the largest of which may be bigger than Ceres), has led astronomers to question exactly how a planet should be defined.

For exoplanets, the question is not whether an object is too small to call a planet (small objects are difficult to detect), but rather whether it is too large. A star maintains itself against gravitational collapse using energy released by nuclear fusion in its interior; only objects at least $7-8 \%$ as massive as our Sun can maintain sufficiently high temperatures in their interiors to become stars. In comparison, the most massive planet in our Solar System, Jupiter, has less than $0.1 \%$ of the mass of the Sun. Various definitions of a planet have been proposed, some based on mass, or the origins of the body, or on its current orbit. The provisional definition adopted by the International Astronomical Union's working group on extrasolar planets is an object that is in orbit about a star and that is smaller than the limit for deuterium fusion to occur (about 13 times the mass of Jupiter).

\section{How to find a planet}

There are several ways to search for exoplanets, and, as planets located many light years away are extremely faint, most methods are indirect - a planet is detected through its influence on the star that it orbits. Different methods are sensitive to different classes of planets and provide complementary information about the planets they find, so most or all of them will contribute to our understanding of the diversity of planetarysystem characteristics.

In 1991, Alexander Wolszczan and Dale Frail announced the presence of two planets in orbit about a pulsar ${ }^{1}$, in what became the first exoplanet discovery. Pulsars are magnetized, rotating neutron stars, which emit radio waves that appear as periodic pulses to an observer on Earth. It was variations in the arrival times of these pulses that signalled the planets' presence to Wolszczan and Frail. The pulse period can be determined very precisely (stable pulsars rank among the most accurate clocks known), and the mean time of pulse arrival at the telescope receiver can be measured especially accurately for rapidly rotating, millisecond pulsars, whose frequent pulses provide an abundance of data. Even though the pulses are emitted periodically, the times at which they reach the receiver are not equally spaced if the distance between the pulsar and the telescope varies in a nonlinear fashion. The
Earth's motion around the Sun and its rotation cause such variations, and these can be calculated and removed from the data. If periodic variations are still present in the data, they may indicate the presence of companion planets orbiting the pulsar.

But by far the most successful planetfinding method at present is the radialvelocity technique. The wavelength of light emitted by distant stars becomes lengthened or shortened depending on whether the star is moving away from or towards the observer (Fig. 2a). By fitting this 'Doppler shift' in the wavelengths of a large number of features within a star's spectrum, the velocity at which the star is moving towards or away from the observer can be precisely measured. Astronomers then subtract the motion of the telescope relative to the centre-of-mass of the Solar System and other known motions, leaving the radial motion of the target star that results from the tug of its own planets. Precise radial-velocity measurements require a large number of spectral lines, and so are not possible for the hottest stars, which have far fewer spectral features than do cooler stars like the Sun. Moreover, stellar rotation and intrinsic variability (including starspots) are major sources of noise for radial-velocity measurements.

There is another slight drawback to both pulsar timing and radial-velocity measurements. Although both are sensitive to the period and the eccentricity of the exoplanet's orbit, an important quantity to measure for any newly discovered planet is its mass. But both methods yield only the product of the planet's mass (divided by that of the star, whose mass can usually be estimated accurately from its spectral characteristics) and the sine of the angle between its orbital plane and the plane of the sky - Msin $i-$ and $i$ usually cannot be determined.

Still, in radial-velocity measurements the leading research groups are now achieving a precision of $3 \mathrm{~m} \mathrm{~s}^{-1}$ on spectrally stable stars (this represents a Doppler shift of 1 part in $\left.10^{8}\right)$. Compare this with our own Solar System: Jupiter causes the Sun's velocity to vary with an amplitude of $12.5 \mathrm{~m} \mathrm{~s}^{-1}$ and a period of 11.86 years; Saturn's effect is the next largest, with an amplitude of $2.7 \mathrm{~m} \mathrm{~s}^{-1}$ and a period of almost 30 years. Thus, with current precision, Jupiter-like planets orbiting Sunlike stars are detectable, although these detections require a long timeline of observations (comparable to the planet's orbital period). Planets smaller than Uranus orbiting very close to stars can also be detected. But finding Earth-like planets in Earth-like orbits is well beyond the capabilities of the radial-velocity technique.

Planets can also be detected from the wobble they induce in the motion of their stars projected onto the plane of the sky (Fig. $2 \mathrm{~b}, \mathrm{c})$. This astrometric technique is most sensitive to massive planets orbiting stars 
that are relatively close to us. But here, because the star's motion is detectable in two dimensions, the planet's actual mass, rather than just the combination Msini, can be measured. Planets in more distant orbits are ultimately easier to detect using astrometry because the amplitude of the star's motion is larger - but then finding these planets requires a longer timeline of observations because of their greater orbital periods.

The detection methods mentioned so far look for a planet's gravitational pull on its star, and so are sensitive to the planet's mass. In contrast, transit photometry detects the amount of starlight that a planet obscures and gives an estimate of the planet's size. If the Earth lies in or near the orbital plane of an extrasolar planet, then, viewed from Earth, that planet periodically blocks a small fraction of the star's light once each orbit. Measuring a star's brightness very precisely can reveal such transits, easily distinguished from other, random effects, such as starspots, by their periodicity and the distinctive, square-well shape of the brightness variation (Fig. 2d). Although this technique detects only the small proportion of planets that happen to line up in this way, thousands of stars can be surveyed within the field of view of one telescope, so transit photometry should be fairly efficient.

Reaching down further into the box of astronomical tricks, we find the microlensing method, which is being used to investigate the distribution of faint, stellar and substellar bodies within our Galaxy². Microlensing arises from the general relativistic bending of the light from a distant star by a massive object (the lens) passing between the source and the observer. Lensing causes the source to appear to brighten gradually to a few times its usual intensity over a period of weeks or months. If the lensing star has planetary companions, then these less massive bodies would produce brief enhancements in the brightness, provided that the line of sight from Earth passes close to the planet. Under favourable circumstances, planets as small as Earth could be detected. But we would only be able to make statistical estimates of the properties of individual planets, and often even of the stars that they orbit, because of the many parameters that influence microlensed light ${ }^{3}$.

So there are many indirect ways to find planets beyond the Solar System, but what about imaging an exoplanet directly? Distant planets are very faint and located near much brighter objects (the star or stars that they orbit), making them extremely difficult to image. The reflected starlight from planets similar in orbit and size to those in our Solar System is roughly only one-billionth as bright as the star, although the contrast decreases a thousandfold for thermal, infrared radiation. Scattering of light by telescope optics and atmospheric variability

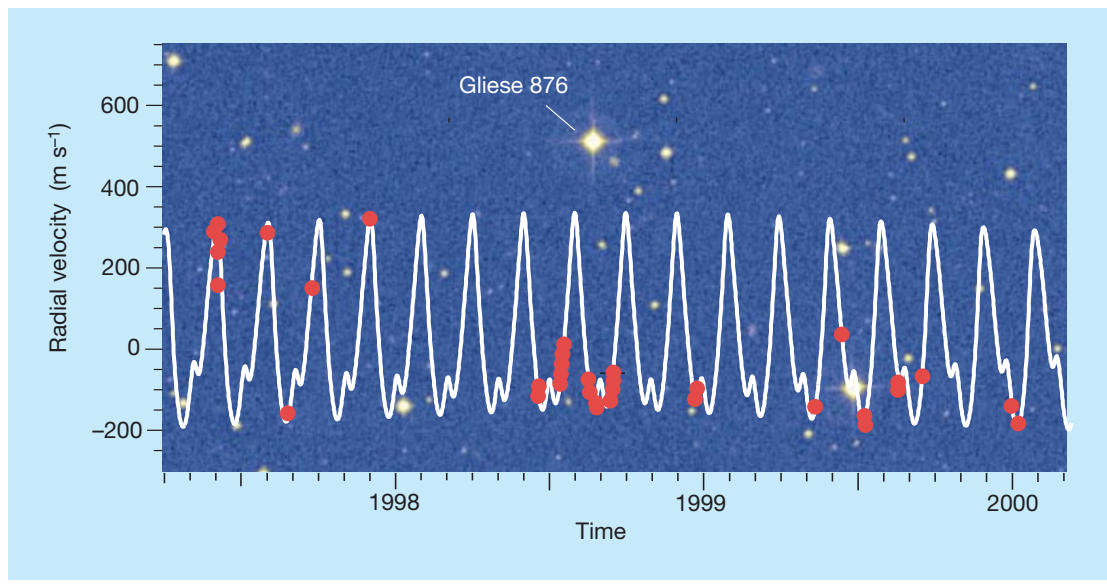

Figure 3 Data from the Keck telescope in Hawaii (red points) show the variation over time of the radial velocity of the star Gliese 876 . The white curve is the best fit to the data points, implying that there are two unseen planets perturbing the motion of the star and each other. (Courtesy E. J. Rivera.)

on Earth add to the difficulty. Nonetheless, technological advances such as adaptive optics should eventually permit imaging and spectroscopic studies of planets orbiting nearby and/or younger, brighter stars.

Ground-based searches using all of these techniques are in progress, and for the future, higher-precision astrometry, transit, microlensing and imaging surveys using spacecraft are being considered. There are still other techniques to explore. Precise timing of the eclipses of eclipsing binary stars has the potential to reveal the masses and orbits of unseen companions. Spectroscopy could be used to identify gases that would be stable in planetary atmospheres but not in stars, and Doppler variations of such signals could yield planetary orbital parameters. Radio emissions similar to those detected from Jupiter could reveal the presence of extrasolar planets. And, of course, artificial signals from an alien civilization could betray the presence of the planets on which they lived (and they might even be willing to provide us with substantially more information).

\section{Observed exoplanets}

Three small planets - the first and the smallest exoplanets known - orbit the pulsar PSR1257+12, a rapidly rotating neutron star around 1.4 times the mass of the Sun and between 2,000 and 3,000 light years from Earth (placing it in our general region of the Milky Way Galaxy, but not a close neighbour even by interstellar standards). The first two to be found have orbital periods of a few months, small eccentricities, and masses a few times as large as the Earth (here we can estimate the actual masses; the star's response to orbital changes produced by mutual gravitational interactions of the planets provides estimates of the masses independent of the orbital inclination). The minimum mass of the inner planet $(M \sin i)$ is only slightly more than that of the
Earth's Moon, and it completes its orbit in just under a month ${ }^{4}$.

So far, all other extrasolar planets for which there is good evidence have been discovered using the radial-velocity (Doppler) technique, although other methods have given tantalizing hints. These exoplanets are much more massive than those around PSR1257+12 and orbit normal, hydrogenburning stars. Most of these stars have masses within a few tens of per cent of that of our Sun and are situated between 20 and 200 light years away — much closer than the pulsar with known planets, but still not our very closest neighbours. The first such exoplanet was discovered ${ }^{5}$ around the star 51 Pegasi (which is slightly less massive than our Sun and a few billion years older) by Michel Mayor and Didier Queloz in 1995. For this planet, Msin $i$ is $45 \%$ of the mass of Jupiter $\left(M_{\mathrm{T}}\right)$ and its orbital period is just 4.23 days less than a twentieth that of Mercury, the closest planet to our Sun. Several similar planets have been found subsequently, implying that about $1 \%$ of Sun-like stars are orbited by Jupiter-like planets with orbital periods of less than one week.

Of the 100 exoplanets now known (with the exception of the pulsar planets), their minimum masses (strictly, $M \sin i$ ) range from about 40 Earth masses (equivalent to $0.12 M_{\mathrm{J}}$, or a bit over twice the mass of Neptune, the third most massive planet in our Solar System) for the planet orbiting the Sun-like star HD 49674, right up to the maximum allowed by the definition of a planet. Most of the observed exoplanets have masses close to (within a factor of a few of) Jupiter's mass, because larger planets are scarcer and smaller ones are more difficult to detect. The only exoplanet so far discovered with a period exceeding that of Jupiter orbits the star 55 Cancri with a 14-year period and $M \sin i=4 M_{\mathrm{J}}$.

The actual size has been measured for only one exoplanet. The close companion to 


\section{Box 1 The Kepler mission}

The detection of Jupiter-like planets generates great scientific and popular interest, but finding planets similar to Earth would be even more exciting. In December 2001, NASA selected the Kepler mission for this task, using transit photometry in space.

Spacecraft are expensive, but there are some observations that just cannot be made from the ground. The effects of the Earth's atmosphere limit photometric precision to roughly $0.1 \%$, which means that although transits by Jupiter-sized planets can be detected from the ground, those by Earth-sized planets cannot ${ }^{10}$. Far greater precision is achievable above the atmosphere, as shown by transit observations of HD 209458 by the Hubble Space Telescope.

With its $0.95-\mathrm{m}$ photometer, Kepler will observe the brightness of 100,000 stars almost continuously for four years, with an extraordinarily large field of view covering
105 square degrees (500 times the area of the Moon). Ultra-highprecision photometry will be achieved by spreading the individual stellar images over dozens of pixels on the device's detectors and by correcting for systematic errors.

The Kepler mission has been designed to be capable of detecting Earth-sized planets around enough stars that a null result would prove that systems like our Solar System are rare. If, on the other hand, our Solar System is typical, Kepler should find about 50 Earth-like planets. This number more than doubles if planets $20 \%$ larger in radius than the Earth are common, and increases many times over if terrestrial planets commonly orbit stars more closely than Mercury does the Sun. On the basis of statistics obtained from radial-velocity surveys, more than 100 inner-orbit, giant planets should be seen in transit, and most of these

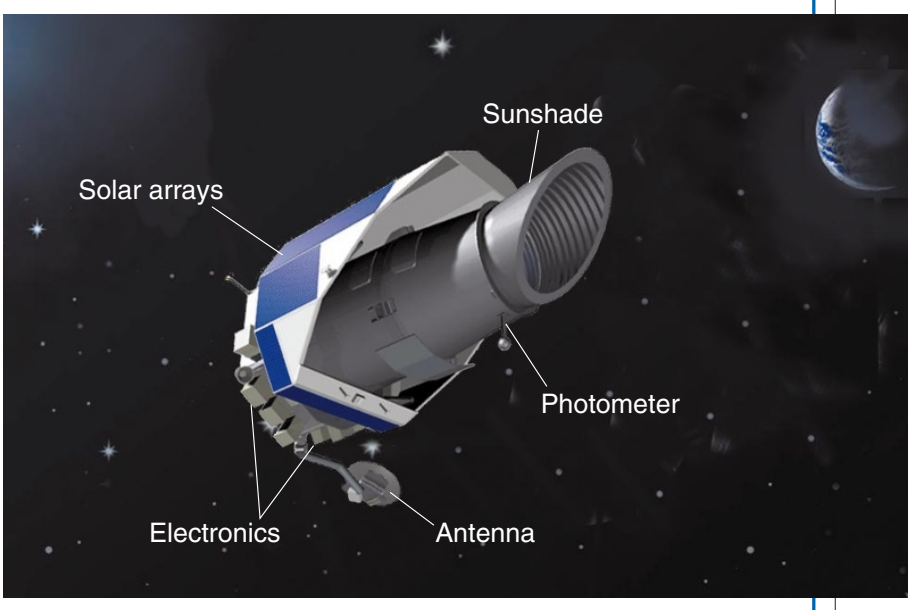

will also be detectable by the variations in light that they reflect from their stars. The masses of many of these inner planets will be determined using radial-velocity measurements.

Kepler's first planet detections dozens of giant planets in inner orbits — should be made within the first two months of launch. But detecting the first true Earth analogues will take 3-4 years of observations, because the orbital periods of such planets are longer and more repetitions of the transits must be observed to separate the signal from the noise in the data. Stay tuned for exciting news at the beginning of the next decade!
HD 209458 (another Sun-like star) was first detected using the Doppler technique but was later observed during transit in front of its star. Measurement of the transit shows that this planet has a radius about 1.35 times that of Jupiter. Moreover, as the tilt of its orbit is known from the transit duration, the planet's actual mass $\left(0.65 M_{\mathrm{J}}\right)$, rather than merely the product $M \sin i$, is known. Together, the mass and size of a planet reveal its density, which implies that the planet orbiting HD 209458 is composed primarily of hydrogen, the lightest and most common element in the Universe, and also the primary constituent of Jupiter and Saturn.

The exoplanetary system of greatest interest to me is that in orbit about Gliese 876. At about one-third the mass of our Sun, this faint red orb is by far the least massive star known to possess any planets. Gliese 876 , only 15 light years from Earth, is also the nearest star for which an exoplanet has unambiguously been found. It is one of less than a dozen stars known to have multiple planets ${ }^{6}$, and the only normal star for which the mutual gravitational perturbations of the planets are clearly evident in the data $^{7,8}$ (Fig. 3)

\section{More to come}

The pace of planet discoveries using radial velocities will continue to increase for at least the next few years, as more observers obtain the spectrometers and the skills to achieve the high Doppler precision required. Smaller inner planets will be detected as precision improves, and planets with longer periods will be found as the data sets grow longer. This should include planets that are true analogues of Jupiter.

Transit photometry, which has already been used to observe the planet orbiting HD 209458 both from the ground and from space, should bear fruit in planet detection in the near future. Several groups are conducting transit searches for close-in giant planets from the ground, mostly using wide-field telescopes with diameters smaller than 25 $\mathrm{cm}$. Both NASA and its European counterpart, ESA, are looking into missions to image Earth-like planets before 2020. A few small telescopes that are designed primarily for studying stellar properties will be launched into space during the next five years and should be able to find planets only a few times the radius of Earth. In 2007, NASA will launch the Kepler mission, which will be capable of detecting true Earth analogues (Box 1). The precision of astrometry from the ground is improving as large interferometers are being built, and even higher-precision astrometry should be achieved from specialpurpose spacecraft, but as astrometry is more sensitive to planets far from stars, observations will need to be conducted for years to observe a full planetary orbit.

With technological advances in the coming years, we shall learn more about how common planets are, and their distributions of size, mass and orbital properties, as well as their densities, colours and atmospheric compositions. We may find that our Solar System, and our own planet, are not that special. But it would be piling speculation on specula- tion to foresee the discovery of life elsewhere.

Although broad classes of future discoveries can be confidently predicted, the particulars cannot. This is because the most successful planet-formation theories are designed to explain the observed properties of planetary systems - the uncertainties in initial conditions, and the complexity of the physics and chemistry of star and planet formation, preclude detailed modelling from first principles ${ }^{9}$. Extrapolation of observed distributions is highly unreliable if the processes creating them are not fully understood. The theorists need more data, and after decades of trying, the observers are now providing a bountiful harvest.

Jack J. Lissauer is in the Space Science Division, NASA Ames Research Center, Moffett Field,

California 94035, USA.

e-mail: jlissauer@ringside.arc.nasa.gov

1. Wolszczan, A. \& Frail, D. Nature 255, 145-147 (1992).

2. Alcock, C. et al. Nature 414, 617-619 (2001).

3. Peale, S. J. Icarus 127, 269-289 (1997).

4. Wolszczan, A. et al. Astrophys. J. 528, 907-912 (2000).

5. Mayor, M. \& Queloz, D. Nature 378, 355-359 (1995).

6. Marcy, G. W. et al. Astrophys. J. 556, 296-301 (2001).

7. Laughlin, G. \& Chambers, J. E. Astrophys. J. 551, L109-L113 (2001).

8. Rivera, E. J. \& Lissauer, J. J. Astrophys. J. 558, 392-402 (2001).

9. Lissauer, J. J. Nature 402, C11-C14 (1999).

10. Gilliland, R. L. et al. Astron. J. 106, 2441-2476 (1993).

\section{Related sites \\ www.obspm.fr/encycl/encycl.html \\ exoplanets.org \\ www.kepler.arc.nasa.gov \\ \ obswww.unige.ch/ udry/planet/planet.html \\ www.astro.psu.edu/users/pspm/arecibo/ \\ planets/planets.html}

